\title{
BMC Endocrine Disorders reviewer acknowledgement 2014
}

Tim Shipley

\section{Contributing reviewers}

The editors of BMC Endocrine Disorders would like to thank all our reviewers who have contributed to the journal in Volume 14 (2014).

Zbigniew Adamczewski
Poland

Amar Agha

Ireland

Nk Agrawal

India

Manuela Albertelli

Italy

Theresa Allain

Malawi

Madson Almeida

Brazil

Alireza Amirbaigloo

Iran

Decio Armanini

Italy

Victor M Baizabal-Aguirre

Mexico

Wei Bao

United States of America

Ilaria Barchetta

Italy

Marisa Bare

Spain

Katharine Barnard

United Kingdom
Analabha Basu

India

Nicholas Beare

United Kingdom

Giorgio Bedogni

Italy

Lucy Ann Behan

Canada

Amaya Belanger-Quintana

Spain

Abdulbari Bener

Qatar

Katrien Benhalima

Belgium

David Beran

Switzerland

Elizabeth Beverly

United States of America

Peyman Björklund

Sweden

Kristien Boelaert

United Kingdom

Marek Bolanowski

Poland

Michael Bonkowski

United States of America
Jérémie Botton

France

Barbara J Boucher

United Kingdom

Jacqueline Boyle

Australia

Monika Buraczynska

Poland

Michael Burch United

Kingdom

Marie-José Butel

France

Atilla Buyukgebiz

Turkey

Milena Caldato

Brazil

Adrian Cameron

Australia

Alessandra Care'

Italy

Manuel Castro Cabezas

Netherlands

Filomena Cetani

Italy

Giriraj Chandak

India

Correspondence: tim.shipley@biomedcentral.com

BioMed Central, Floor 6, 236 Gray's Inn Road, London WC1X 8HB, UK 


\author{
Yi-Cheng Chang \\ Taiwan \\ Yuan-Jia Chen \\ China \\ Shih-Ping Cheng \\ Taiwan \\ Ching-Ju Chiu \\ Taiwan \\ Gabriel Chodick \\ Israel \\ Mary Chong \\ Singapore \\ Dirk Lund Christensen \\ Denmark \\ Pm Clark United \\ Kingdom \\ Angela Clow \\ United States of America \\ Neale Cohen \\ Australia \\ Bernard Conte-Devolx \\ France \\ Flavia Costa- Barbosa \\ Brazil \\ Rachel Crowley \\ Ireland
}

Alberto Da Silva Moraes

Brazil

Eleonora Dalla Bella

Italy

Shannon Davis

United States of America

Giovambattista Desideri Italy

Thomas Dickmeis

Germany

George Dimitriadis

Greece

Sima Djalali

Switzerland

Horacio Domené

Argentina

Periklis Dousdampanis

Greece
Koen Dreijerink

Netherlands

Juan Duenas

Colombia

Carlos S Duque

Colombia

Peter Ebeling

Australia

Roberto Esposito

Italy

Denis Evoy

Ireland

Oana Falup-Pecurariu

Romania

Fabio Faucz

United States of America

E. Vincent Faustino

United States of America

Huai Feng

United States of America

Fabio Fernandes-Rosa

France

Bruno Ferraz-De-Souza

Brazil

Charles Fox United

Kingdom

Chris Frampton New

Zealand

Davide Francomano

Italy

Yoshio Fujioka

Japan

Filip Gabalec

Czech Republic

Roger Gadsby

United Kingdom

Claudia Gagnon

Canada

Neetika Garg

United States of America

Erica Gentilin

Italy

Peter Gergics

United States of America
Laura Gianotti

Italy

Oliver Gimm

Sweden

Paul Glynn

United Kingdom

Mukesh Gohel

India

Cumali Gokce

Turkey

Larissa Gomes

Brazil

Elise Gomez-Sanchez

United States of America

Margaret Grey

United States of America

Maria Rosaria Gualano

Italy

Martin Gulliford

United Kingdom

Vipin Gupta

India

Rubina Hakeem

Pakistan

Thang Han

United Kingdom

Nihal Hatipoglu

Turkey

Jan Willem Haveman

Netherlands

Weimin He

China

Ian Holdaway

New Zealand

Deborah Jane Holmes-Walker

Australia

Fei-Hsiu Hsiao

Taiwan

Peter Igaz

Hungary

Richard Ijzerman

Netherlands

Romaina Iqbal

Pakistan 
Somchit Jaruratanasirikul

Thailand

Michelle Jones

United States of America

Prapaporn Jungtrakoon

United States of America

Gregory Kaltsas

Greece

Zuleyha Karaca

Turkey

Anuradhani Kasturiratne

Sri Lanka

Prasad Katulanda

Sri Lanka

\section{Atsunari Kawashima \\ Canada}

Fahrettin Kelestimur

Turkey

Marcus Kennedy

Ireland

Anuradha Khadilkar

India

Muhammad Kibriya

United States of America

Dong-Jun Kim

Korea, South

Christian Koch

United States of America

Josef Köhrle

Germany

Alexander Kokkinos
Greece

Michiharu Komatsu

Japan

Evangelos Kontopantelis

United Kingdom

Juraj Koska

United States of America

Nermin Kösüs

Turkey

James Krinsley

United States of America

Grace Marie Ku

Philippines
Ganesh Kumar

United States of America

Tommy Kyaw-Tun

Ireland

George Kyriazis

United States of America

Stefano La Rosa

Italy

Pauline Siew Mei Lai

Malaysia

Vaia Lambadiari

Greece

Lisa Langsetmo

Canada

Mary Larkin

United States of America

Anna Lauber-Biason

Switzerland

David Lebeaux

France

Duk-Hee Lee

Korea, South

Antonio Lerario

Brazil

Jianhong Li

China

Xiaoying Li

Canada

Stavros Liatis

Greece

Rossella Libe

France

Suh-Jen Lin

United States of America

Yijia Lou

Comoros

Paul Lucassen

Netherlands

Naim Maalouf

United States of America

Sujay Madduri

United States of America

Dianna Magliano

Australia
Michele Malaguarnera

Italy

Rayaz Malik

United Kingdom

Ashwini Mallappa

United States of America

Robert Mallet

United States of America

Jessica Markowitz

United States of America

Vincenzo Marotta

Italy

Raman Marwaha

India

Melinda Maryniuk

United States of America

Paolo Marzullo

Italy

Rocio Mateo-Gallego

Spain

Sandeep K Mathur

India

Gherardo Mazziotti

Italy

Ciara Mcdonnell

Ireland

Malachi Mckenna

Ireland

Mark Mclean

Australia

Diane Mege

France

Nicholas Mezitis

United States of America

Teresa Mezza

Italy

Paras Kumar Mishra

United States of America

Marie Misso

Australia

Maureen Monaghan

United States of America

Carla Moran

United Kingdom 
Yuji Moriwaki
Japan

Thomas Mueller

Austria

Arijit Mukhopadhyay

India

Giovanna Muscogiuri

Italy

Parimala Narne

India

Michael Nauck

Germany

Juan Nicola

Argentina

Michael O'Reilly

United Kingdom

Zeynep Osar Siva

Turkey

Tríona O'Shea

Ireland

Nikolaos Papanas

Greece

Renato Pasquali

Italy

Klara Paudel

United Kingdom

Andre Peinnequin

France

Phillip Pekala

United States of America

M. Yanina Pepino

United States of America

Luigi Petramala

Italy

Elzbieta Petriczko

Poland

Daniel Petrovic

Slovenia

Gustavo Duarte Pimentel

Brazil

Chang Ping'An

China

Milan Piya

United Kingdom
Giuseppe Procopio

Italy

Manuel Puig-Domingo

Spain

Fanny Rancière

France

Jonas Ranstam

Sweden

Ulrich Renner

Germany

Musarrat Riaz

Pakistan

Evangelos Rizos

Greece

Karine Rizzoti

United Kingdom

Jo-Anne Rizzotto

United States of America

Edna Roche

Ireland

Vincenzo Rochira

Italy

Santiago Rodriguez-Segade

Spain

William Rojas

Colombia

Vanessa Ronconi

Italy

Gian Paolo Rossi

Italy

Mario Rotondi

Italy

Marek Ruchala

Poland

R.A.C. Ruiter

Netherlands

Isabelle Runkle De La Vega

Spain

Banshi Saboo

India

Matteo Santoni

Italy

Vinod Scaria

India
Carlos Alberto Scrideli

Brazil

Teresa Maria Seccia

Italy

Alissa Segal

United States of America

Yutaka Seino

Japan

Arundhati Sharma

India

Mark Sherlock

Ireland

Cephas Sialubanje

Zambia

Diarmuid Smith

Ireland

Miguel A Sogorb

Spain

Martin Soubrier

France

Carole Spencer

United States of America

Seamus Sreenan

Ireland

Tobias Stalder

Germany

Douglas Steinke

United Kingdom

Jan Stepan

Czech Republic

Joanna Stewart

New Zealand

Monique Stone

Australia

Michael Stowasser

Australia

Alexios Strimpakos

Greece

Elsa Strotmeyer

United States of America

Gonca Tamer

Turkey

Nagaaki Tanaka

Japan 
Molly Tanenbaum

United States of America

Francesco Tassone

Italy

Nicholas Tentolouris

Greece

Ajay Thankamony

United Kingdom

Jerome Thevenot

Finland

\section{Sathish Thirunavukkarasu}

Australia

Debbie Thompson

Jamaica

Rodrigo Toledo

United States of America

Jeremy Tomlinson

United Kingdom

Jorg Tost

France

Ericka Trarbach

Brazil

Panagiotis Tsapogas

Greece

Toshihiko Tsukada

Japan

Tiinamaija Tuomi

Finland

Kultigin Turkmen

Turkey

Mariko Uehara

Japan
Zoltan Ungvari

United States of America

Vytautas Usonis

Lithuania

Mukremin Uysal

Turkey

Tone Gretland Valderhaug

Norway

Katherine Valeros

Singapore

André Van Beek

Netherlands

Danielle Van Der Kaay

Canada

Aj Van Der Lely

Netherlands

Tom Van Herpe

Belgium

Emerielle Vanzela

Brazil

Dimitra Argyro Vassiliadi

Greece

Srividya Vasu

United Kingdom

Alejandro Velez Hoyos

Colombia

Kavita Venkataraman

Singapore

Giuseppe Vezzoli

Italy

Mary Vouyiouklis

United States of America
Xiangbing Wang

United States of America

Susan Webb

Spain

Jan-Maarten Wit

Netherlands

Viroj Wiwanitkit

Thailand

Wenxun Wu

China

Yuichiro Yamada

Japan

Kazuki Yasuda

Japan

Xingwang Ye

China

Ozcan Yildiz

Turkey

Danxia Yu

United States of America

Carles Zafon

Spain

Raul Zamora Ros

France

Yan Zheng

United States of America

Received: 14 January 2015 Accepted: 14 January 2015

Published: 12 February 2015

doi:10.1186/1472-6823-15-3

Cite this article as: Shipley: BMC Endocrine

Disorders reviewer acknowledgement 2014.

BMC Endocrine Disorders 2015 15:3. 